\title{
Structural and Compositional Effects in Epitaxially-Strained Vanadate Thin Films
}

\author{
Duncan T.L. Alexander ${ }^{1 *}$, Hugo Meley ${ }^{2}$, Bernat Mundet ${ }^{1,2}$, Jean-Marc Triscone ${ }^{2}$ and Stefano Gariglio ${ }^{2}$ \\ 1. Electron Spectrometry and Microscopy Laboratory (LSME), Institute of Physics (IPHYS), Ecole \\ Polytechnique Fédérale de Lausanne (EPFL), Lausanne, Switzerland. \\ 2. Department of Quantum Matter Physics (DQMP), University of Geneva, Geneva, Switzerland. \\ * Corresponding author: duncan.alexander@epfl.ch
}

Breaking the inversion symmetry in artificial hetero-structures is proving to be a powerful approach to create novel quantum states, thereby providing new functionalities with potential for the development of revolutionary devices. Based on this, a way to achieve "hybrid improper ferroelectricity" in perovskite superlattices was recently devised by ab-initio theory $[1,2]$. The working principle rests on the stacking of odd numbers of orthorhombic distorted $A B \mathrm{O}_{3}$ and $A^{\prime} \mathrm{BO}_{3}$ unit cells in order to generate a macroscopic polarization out of the uncompensated displacements of the $A$ site cations in the superlattice. These displacements are the result of the strong coupling between chemistry and structure in perovskite oxides. Towards this goal, here we investigate $A \mathrm{VO}_{3}$ epitaxial thin films grown under different conditions by pulsed laser deposition, where $A$ is either La or Pr, using aberration-corrected scanning transmission electron microscopy (STEM) to identify local strain-induced structural and compositional effects.

The films are studied using a double aberration-corrected Titan Themis 60-300 operated at $300 \mathrm{kV}$, using a $20 \mathrm{mrad}$ convergence semi-angle. High angle annular dark field (HAADF) and annular bright field (ABF) image series are simultaneously recorded, typically at consecutive scans rotations of $90^{\circ}$, using Fischione and Gatan photomuliplier tube detectors respectively. Post-acquisition, effects of scan drift and other distortions are compensated via rigid and non-rigid alignment using Smart Align [3]. Chemical analyses are made with energy dispersive X-ray spectroscopy, using ChemiSTEM detectors and Velox software, and electron energy-loss spectroscopy (EELS), using a Gatan GIF Quantum ERS.

In a first step, strain effects on the crystal lattice are studied by analyzing films of $\mathrm{LaVO}_{3}$ grown under either biaxial compression (on $\left.\left(\begin{array}{lll}0 & 0 & 1\end{array}\right) \mathrm{SrTiO}_{3}\right)$ or tension (on $\left.\left(\begin{array}{lll}1 & 1 & 0\end{array}\right)_{O} \mathrm{DyScO}_{3}\right)$. In both cases, the atomic structure of the bulk film matches well that predicted by ab-initio density functional theory calculations, with an antipolar $X_{5}^{-}$mode clearly visible as $15-20 \mathrm{pm}$ displacements of La cations, either perpendicular to the substrate plane for $\mathrm{SrTiO}_{3}$, or parallel to it for $\mathrm{DyScO}_{3}$ [4]. However, while this mode starts directly at the film/substrate interface on $\mathrm{SrTiO}_{3}, \mathrm{STEM}$ measurements reveal an unexpected "transition layer" (TL) structure along the film/substrate interface of the films on $\mathrm{DyScO}_{3}$, when imaged on the $\left[\begin{array}{lll}1 & 1 & 0\end{array}\right]_{P C}$ zone axis ( $P C$ : pseudo-cubic). While compositional measurements identify that this TL is chemically identical to the film bulk, the TL, which is uniformly 9-10 $P C$ unit cells thick, gives a strain or phase contrast which render it visible in HAADF images. Ongoing work to characterize this TL suggests that its structure is more similar to that of the $\mathrm{DyScO}_{3}$ than the bulk $\mathrm{LaVO}_{3}$ film, for instance both in $A$-site cation positions and in a broadening of the La atom column intensity on an axis parallel to the $0.6 \AA$-separated Dy dumbbells in the substrate; see Figure 1. Theoretical concepts to explain the TL formation based around the connectivity of the $\mathrm{O}$ octahedra from substrate to film are being developed.

In a second step, we show preliminary studies of multilayers films $\sim 20 \mathrm{~nm}$ thick of $\left(\mathrm{LaVO}_{3}\right) n /\left(\mathrm{PrVO}_{3}\right) n$ repetitions, for $3 \leq n \leq 8$ where $n$ is in terms of $P C$ unit cells. Because X-ray diffraction structure measurements average over the film thickness, STEM imaging is vital to identify changes in charge 
ordering between the layers that could lead to improper ferroelectricity. The STEM imaging shows that all of the films have an in-plane $X_{5}^{-}$mode, visible on a $\left[\begin{array}{lll}1 & 1 & 0\end{array}\right]_{P C}$ zone axis. Remarkably, compositional analyses also identify a different chemical behavior between the films with $n=$ even and $n=$ odd. Specifically, those with $\mathrm{n}=$ odd - which are also the candidates to show improper hybrid ferroelectricity - show a strong intermixing of La and Pr cations between the different layers, while those with $n=$ even are much more compositionally distinct; see Figure 2. We are engaged in efforts to understand the reason for this phenomenon, which could have strong implications for this field [5].

References:

[1] J M Rondinelli and C J Fennie, Adv. Mater. 24 (2012), p. 1961.

[2] J Varignon et al., Sci. Rep. 5 (2015), 15364.

[3] L Jones et al., Adv. Struct. Chem. Imaging 1 (2015), 8.

[4] H Meley et al., APL Materials 6 (2018), 046102.

[5] This work was supported by the Swiss National Science Foundation - division II, by the Synergia Project No. 154410. We acknowledge the CIME, EPFL for access to its facilities; at the CIME we thank Colette Vallotton, Dr Barbora Bartová and Danièle Laub for TEM specimen preparation.
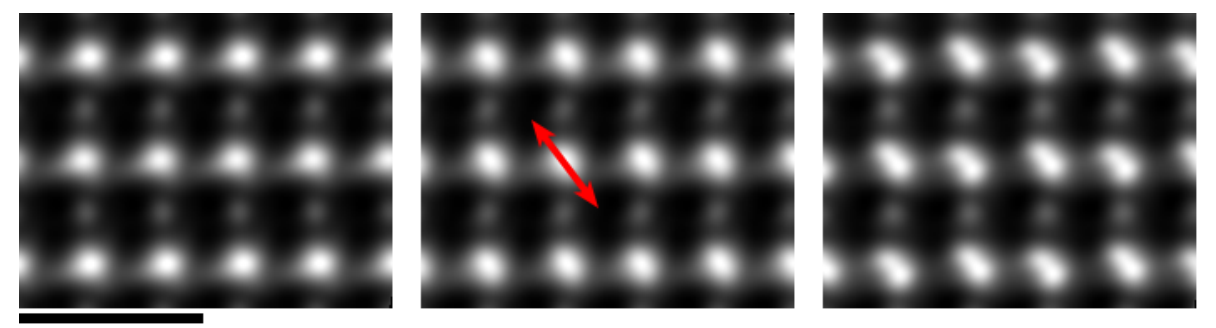

Figure 1. Averaged HAADF images of the atomic structure of the bulk $\mathrm{LaVO}_{3}$ (left), the $\mathrm{LaVO}_{3} \mathrm{TL}$ (middle), and $\mathrm{DyScO}_{3}$ substrate (right) extracted from one larger image using a template matching and alignment process, on the $\left[\begin{array}{lll}1 & 1 & 0\end{array}\right]_{P C}$ zone axis. Scale bar: $0.5 \mathrm{~nm}$. In the TL, the brighter La columns are elongated by $\sim 20 \mathrm{pm}$ on the axis indicated by the red arrow, as compared to those in the bulk structure; this elongation is parallel to the axis of the $0.6 \AA$ atom column separation Dy dumbbells in the substrate.
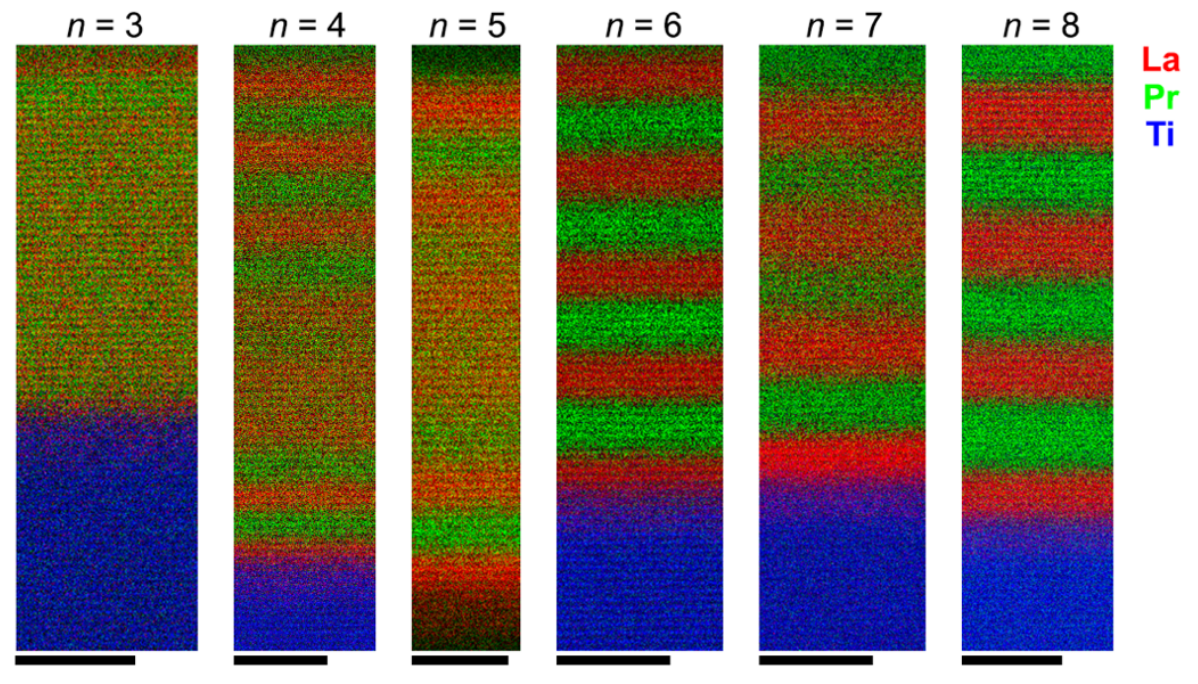

Figure 2. STEM-EELS compositional maps for La (red), $\mathrm{Pr}$ (green), Ti (blue) of $\left(\mathrm{LaVO}_{3}\right) n /\left(\mathrm{PrVO}_{3}\right) n$ multilayers on $\mathrm{SrTiO}_{3}$ substrates going from $n=3$ to $n=8$. Scale bars: $5 \mathrm{~nm}$ 